\title{
Proposta de adequação da arquitetura do AVA Openredu para suporte a técnicas de análise quantitativa de dados educacionais
}

\author{
Juliano C.T. Vaz ${ }^{1}$, Raphael A. Dourado ${ }^{1}$, Alex S. Gomes ${ }^{1}$,Rodrigo L. Rodrigues ${ }^{2}$, \\ Tiago J.D.D. Nogueira1, Sylvio E. Freire ${ }^{1}$ \\ ${ }^{1}$ Centro de Informática - Universidade Federal de Pernambuco (UFPE) \\ ${ }^{2}$ Departamento de Educação - Universidade Federal Rural de Pernambuco (UFRPE) \\ \{jctv, rasd2, asg, tjddn, sef\} @cin.ufpe.br, rlreded.ufrpe.br
}

Resumo. Este trabalho propõe uma adequação para o Ambiente Virtual de Aprendizagem (AVA) Openredu para suportar técnicas de análise quantitativa de dados tais como Mineração de Dados Educacionais (MDE) e Learning Analytics (LA) a partir de um conjunto de requisitos de dados identificados em estudos anteriores. Os resultados obtidos foram (1) a remodelagem da arquitetura de dados da plataforma, que tem como objetivo possibilitar diversas aplicações com os dados armazenados, e sendo uma destas a visualização de dados associados, foi desenvolvido (2) um painel analítico.

\begin{abstract}
This paper proposes an adequation of the Learning Management System (LMS) Openredu to support quantitative data analysis techniques such as Educational Data Mining (EDM) and Learning Analytics (LA) based on a set of data requirements identified in previous studies. The contributions of this study are (1) the redesign of the platform's data architecture, which has the objective of making possible several applications with the stored data, and being one of them the associated data visualization, was designed (2) a dashboard.
\end{abstract}

\section{Introdução e Fundamentação Teórica}

A Internet, no contexto do e-learning e do Ensino a Distância (EAD), vem permitindo o uso cada vez mais frequente de Ambientes Virtuais de Aprendizagem (AVA). Estes armazenam um grande volume de dados relativos às interações dos aprendizes nos AVAs e podem ser utilizados como insumo para diversos tipos de análise capazes de extrair informações sobre os processos de aprendizagem, dificuldades e comportamento de cada estudante [Ray \& Saeed 2018].

Segundo Silva et al. (2017), no campo da Ciência dos Dados Educacionais (CDE), a Mineração de Dados Educacionais (MDE) e o Learning Analytics (LA) são as áreas que têm como similaridade a maneira com que os dados são analisados e diferenciando-se na abordagem de cada tipo de problema. A MDE busca tratar e processar os dados, enquanto o LA se concentra em análises de dados. Nesse contexto, Paiva et al. (2018) afirmam que, nesse ramo, há necessidade de mecanismos que favoreçam novas formas de visualizar diferentes informações, de modo que contribuam para a obtenção de insights a partir dos dados. Logo, a Visualização de Dados, que busca fornecer representações visuais de conjuntos de dados para ajudar as pessoas a realizar tarefas com mais eficiência [Munzner 2014], junto às demais áreas, originaram a área de pesquisa chamada Visual Learning Analytics [Vieira, Parsons \& Byrd 2018].

Nesse contexto, este trabalho propõe, a partir de uma revisão de literatura e consulta a especialistas, uma proposta de adequação do AVA Openredu [Gomes et al. 2012] para a incorporação de técnicas de análise quantitativa de dados educacionais. O restante deste artigo está organizado da seguinte forma: a Seção 2 descreve a metodologia utilizada; a Seção 3 
apresenta os resultados obtidos; e a Seção 4 aponta as conclusões deste estudo e trabalhos futuros.

\section{Método}

O desenvolvimento deste projeto se deu a partir do seguinte percurso metodológico: (1) revisão narrativa da literatura [Okoli \& Schabram 2010]; (2) identificação dos requisitos de dados em Ambientes Virtuais de Aprendizagem (AVAs) para a aplicação de técnicas de análise quantitativa de dados educacionais, a partir de pesquisa bibliográfica, entrevistas e consulta a especialistas na área; (3) elaboração de um documento de requisitos com recomendações sobre qual conjunto de variáveis deve idealmente ser armazenado por AVA para potencializar o uso de MDE e LA; e (4) avaliação da qualidade dos requisitos elicitados por meio da adequação da arquitetura do AVA — Openredu — para atendê-los.

\section{Resultados e Discussão}

Esta seção apresenta os três resultados alcançados no projeto: identificação dos requisitos de dados em AVAs; definição de uma arquitetura de dados para a plataforma utilizada como caso de uso e prototipação de um dashboard para visualização das variáveis identificadas. Quanto a arquitetura e o dashboard, ambos continuam em desenvolvimento e já são funcionais. As subseções a seguir descrevem estes resultados em detalhes.

\subsection{Identificação dos requisitos de dados}

A partir de uma revisão narrativa de literatura e com base na opinião de especialistas foi selecionado um conjunto inicial de trabalhos na área de MDE e LA composto por revisões sistemáticas de literatura, teses de doutorado e pesquisas exemplares para a identificação dos requisitos de dados. Apesar da seleção dos requisitos identificados ser detalhada na publicação em Vaz et al. (2018), vale salientar que a granularidade de dados desses requisitos podem, em diferentes escalas, passar por desmembramentos, possibilitando variáveis mais específicas para uma proposta de estudo aplicado, além disso, proporcionando doravante e em uma perspectiva longitudinal, uma grande base de dados (datawarehouse) para qualquer AVA que seguir os apontados apresentados no artigo publicado. Com base em algumas das variáveis deste conjunto - as existentes na modelagem de dados atual do Openredu - foi elaborado um modelo de representação gráfica para cada uma delas, culminando no protótipo de interface descrito na Seção 3.3 .

\subsection{Arquitetura do Openredu Analytics}

A arquitetura do Openredu Analytics (subsistema de logging) foi elaborada com especialistas e colaboradores na área envolvidos no projeto. Ademais, a função da arquitetura de um software não é criar um padrão a ser seguido por toda a vida do sistema, mas deixa possibilidades para evolução do sistema, vendo que não existe garantia sob qual a proporção de mudanças que uma regra de aplicação pode alterar no futuro. Sendo assim, para o Model-view-presenter (MVP) [Lappalainen \& Kobayashi 2017] da aplicação temos o cenário mostrado na Figura 1, no qual as instâncias do Openredu (Redu A, B e C) enviam os $\log s$ dos eventos - registro das interações do usuário com a plataforma - para o RES (Redu Event Store), responsável pelo registro das instâncias e persistência dos seus eventos. O RES, expondo uma REST API, recebe os logs para armazenamento dos eventos de sua forma mais elementar, podendo estes serem usados futuramente em novas aplicações.

A Analytics API consultará os eventos no RES, fazendo um pré-processamento e alimentando as aplicações de machine learning, data mining, data visualization, data analysis etc (representados como os círculos sobre a Analytics API), persistindo os resultados. Nesse caso, o dashboard é uma dessas aplicações possíveis, na Figura 1 chamado de Dash. É a interface que apresentará os relatórios e visualizações dos dados consolidados no banco da 
Analytics API. O Dash utiliza a Analytics API para obter os dados, sendo que a consulta dos eventos ocorrerá uma vez por dia, onde o RES deve retornar apenas os eventos que acontecem nas últimas 24 horas.

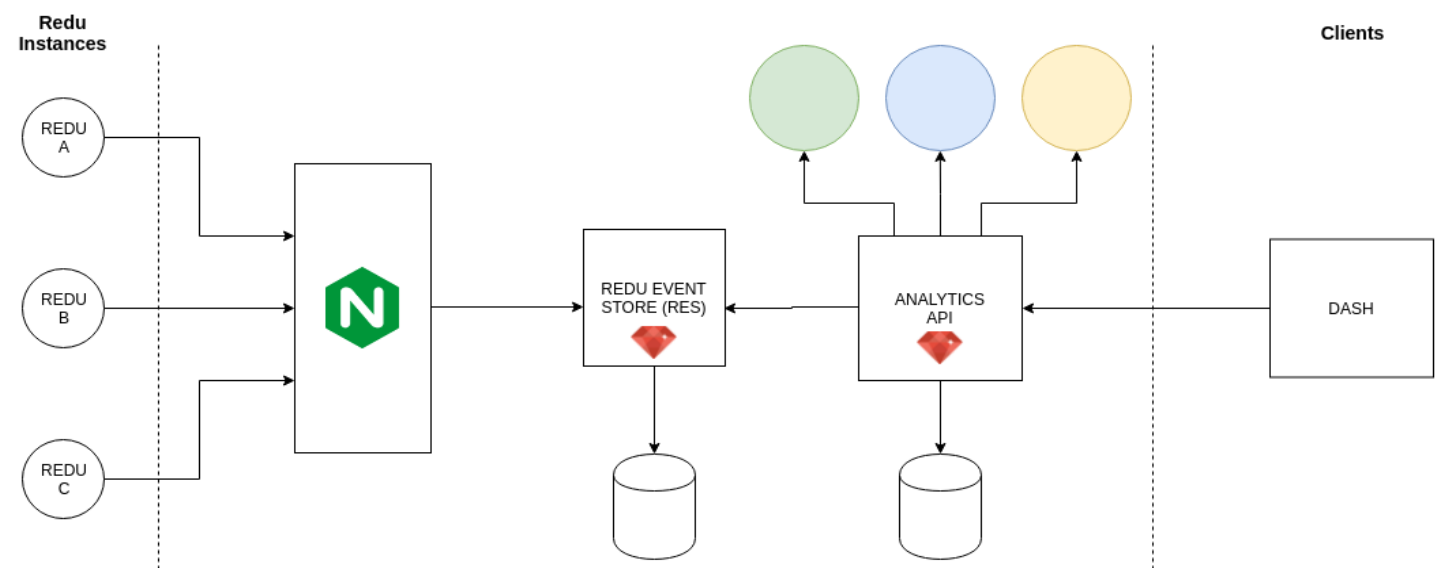

Figura 1. Arquitetura proposta para o Openredu Analytics

Já o processo de consolidação pode ser feito localmente na própria aplicação ou em algum serviço externo (por exemplo, Python ou R). Este será o funcionamento final no qual os resultados obtidos ou modelo são interpretados e usados para tomar decisões sobre o ambiente educacional.

\subsection{Dashboard (painel analítico)}

A partir de consulta a especialistas e colaboradores, análise de padrões de design para esse tipo de trabalho e usando técnicas de prototipação de baixo nível de fidelidade, foi concebida a interface do painel analítico. Posteriormente, foi realizada prototipação de alta fidelidade, resultando no protótipo mostrado na Figura 2 que exibe gráficos desenvolvidos, sendo alguns deles: atividades acessadas, tipo de comentários e recursos acessados. Nesta mesma figura há uma sidebar (à esquerda) com um filtro - menu de links mais específicos - para a manipulação da granularidade da representação dos dados com uma hierarquia acerca do nível da arquitetura da informação do Openredu do que se deseja visualizar, além da definição do recorte de período (datas de início e fim) e do componente de configuração do usuário na parte superior direita.

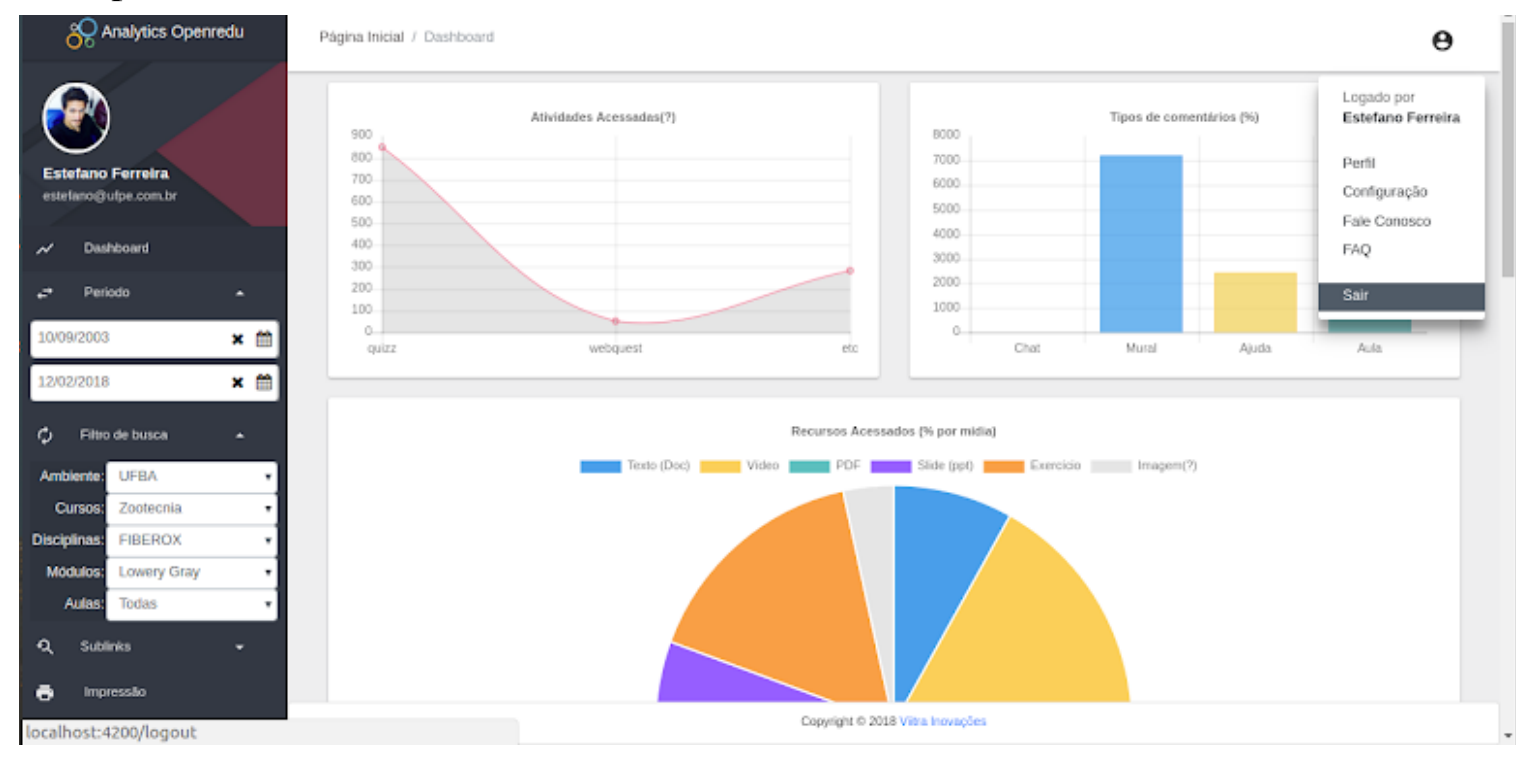

Figura 2. Homepage proposta para o dashboard 


\section{Conclusões}

Este trabalho apresentou uma proposta de adequação da arquitetura de um AVA - Openredu para garantir suporte a técnicas de análise quantitativa de dados educacionais, mas serve como modelo para qualquer AVA que deseje garantir tal suporte. Partindo de estudos anteriores sobre requisitos de dados em MDE e LA, foi realizada a remodelagem da arquitetura de dados da plataforma Openredu e prototipação de um painel de visualização de dados. Os mesmos foram desenvolvidos e continuam em aprimoramentos funcionais.

Dentre os trabalhos futuros suscitados por esta pesquisa estão: a verificação da arquitetura e do dashboard desenvolvidos com criação de testes funcionais para consolidá-los; a validação dos resultados com especialistas em Ciência de Dados Educacionais - CDE; a aplicação de um protocolo de revisão sistemática para garantir maior rigor na seleção dos trabalhos utilizados para compilar os requisitos; e a comparação com trabalhos relacionados sistematizando o que já foi feito nessa área para auxiliar a análise de dados educacionais.

\section{Agradecimentos}

Os autores agradecem ao apoio financeiro da FACEPE (processos No.0525-1.03/14, BCT-0016-1.03/15 e BIC-1303-1.03/17) e do CNPq (processos 310.466/2012-1, 475634/2013-6, 307202/2015-1 e 140973/2017-6) e do apoio da SEAD/UNIVASF por colaborar e oferecer o ambiente para a pesquisa.

\section{Referências}

Okoli, C., \& Schabram, K. (2010). "A Guide to Conducting a Systematic Literature Review of Information Systems Research". Sprouts: Working Papers on Information Systems, 10(26).

Gomes, A. S., Rolim, A. L., \& Silva, W. M. (2012). "Educar com o Redu”. Recife: Redu Educational Technology.

Lappalainen, S., \& Kobayashi, T. (2017). “A Pattern Language for MVC Derivatives". Disponível em: < https://goo.gl/dqGTmg>. Acessado em 10 de fevereiro de 2019, p 1-5.

Munzner, T. (2014). "Visualization Analysis and Design". CRC Press.

Paiva, R., Bittencourt, I. I., Lemos, W., Vinicius, A., \& Dermeval, D. (2018). "Visualizing Learning Analytics and Educational Data Mining Outputs", In International Conference on Artificial Intelligence in Education p. 251-256.

Silva, L. A., Silveira, I. F., Silva, L., Rodrigues, R., \& Ramos, J. L. C. (2017). "Ciência de Dados Educacionais: definições e convergências entre as áreas de pesquisa". In Workshops do Congresso Brasileiro de Informática na Educação. p. 764-773.

Ray, S. \& Saeed, M. (2018). “Applications of Educational Data Mining and Learning Analytics Tools in Handling Big Data in Higher Education”. In Applications of Big Data Analytics, p. 135-160.

Vaz, J. C. T., Dourado, R., Gomes, A., \& Rodrigues, R. (2018). "Requisitos de Dados para uso de Técnicas de Análise Quantitativa de Dados Educacionais em AVAs". In Simpósio Brasileiro de Informática Na Educação - SBIE, 29(1), p. 1941-1945.

Vieira, C., Parsons, P. \& Byrd, V. (2018). "Visual learning analytics of educational data: A systematic literature review and research agenda". In Computers \& Education, p. 119-135. 